\title{
Towards sensitive terahertz detection via thermoelectric manipulation using graphene transistors
}

\author{
Changlong Liu' ${ }^{1,2}$, Lei Du ${ }^{1,2}$, Weiwei Tang ${ }^{1,2}$, Dacheng Wei ${ }^{3}$, Jinhua Li ${ }^{4}$, Lin Wang ${ }^{1,2,5}$, Gang Chen ${ }^{1,2}$,
} Xiaoshuang Chen ${ }^{1,2,5}$ and Wei Lu ${ }^{1,2,5}$

\begin{abstract}
Graphene has been highly sought after as a potential candidate for hot-electron terahertz ( $\mathrm{THz}$ ) detection benefiting from its strong photon absorption, fast carrier relaxation, and weak electron-phonon coupling. Nevertheless, to date, graphene-based thermoelectric $\mathrm{THz}$ photodetection is hindered by low responsivity owing to relatively low photoelectric efficiency. In this work, we provide a straightforward strategy for enhanced THz detection based on antenna-coupled CVD graphene transistors with the introduction of symmetric paired fingers. This design enables switchable photodetection modes by controlling the interaction between the $\mathrm{THz}$ field and free hot carriers in the graphene-channel through different contacting configurations. Hence a novel "bias-field effect" can be activated, which leads to a drastic enhancement in $\mathrm{THz}$ detection ability with maximum responsivity of up to $280 \mathrm{~V} / \mathrm{W}$ at 0.12 $\mathrm{THz}$ relative to the antenna area and a Johnson-noise limited minimum noise-equivalent power (NEP) of $100 \mathrm{pW} / \mathrm{Hz}^{0.5}$ at room temperature. The mechanism responsible for the enhancement in the photoelectric gain is attributed to thermophotovoltaic instead of plasma self-mixing effects. Our results offer a promising alternative route toward scalable, wafer-level production of high-performance graphene detectors.
\end{abstract}

\section{Introduction}

Recently, two-dimensional (2D) materials (transition metal dichalcogenides (TMDs), graphene, black phosphorus, etc.) have ignited intensive interest due to their novel electronic and photonic properties, which are distinct from those of their bulk counterparts, such as defectfree topological transport ${ }^{1-3}$, linear dichorism ${ }^{4,5}$, superconductivity $^{6,7}$, and plasmonics ${ }^{8,9}$. It has been proved experimentally that few-layer 2D materials can strongly

Correspondence: Lin Wang (wanglin@mail.sitp.ac.cn) or

Gang Chen (gchen@mail.sitp.ac.cn) or Xiaoshuang Chen (xschen@mail.sitp.ac. cn)

${ }^{1}$ State Key Laboratory of Infrared Physics, Shanghai Institute of Technical Physics, Chinese Academy of Sciences, 500 Yutian Road, Shanghai 200083,

China

${ }^{2}$ University of Chinese Academy of Sciences, No 19 A Yuquan Road, Beijing 100049, China

Full list of author information is available at the end of the article

These authors contributed equally: Changlong Liu, Lei Du, Weiwei Tang. interact with light ${ }^{10,11}$, which indicates they are promising materials with great potential in optoelectronic devices for communication, sensing, and imaging, particularly in the visible and near-infrared range. However, it is rather difficult to extend the spectral response of $2 \mathrm{D}$ materials to the mid-/far-infrared and terahertz $(\mathrm{THz})$ regime owing to the relatively large bandgap of these materials. Many efforts, such as bandgap engineering and heterogeneous integration with quantum dots or other 2D materials (van der Waals heterostructures), have been dedicated to resolving this bottleneck ${ }^{12-14}$. Nevertheless, light absorption in narrow-gap 2D materials such as TMDs decreases significantly when the photon energy is far below the interband threshold, similar to the behavior of other bulk semiconductors.

On the other hand, gapless graphene, in principal, can interact strongly with electromagnetic fields over a much wider spectral range. The photoresponsivity of graphene

\section{(c) The Author(s) 2018}

(c) Open Access This article is licensed under a Creative Commons Attribution 4.0 International License, which permits use, sharing, adaptation, distribution and reproduction cc) in any medium or format, as long as you give appropriate credit to the original author(s) and the source, provide a link to the Creative Commons license, and indicate if changes were made. The images or other third party material in this article are included in the article's Creative Commons license, unless indicated otherwise in a credit line to the material. If material is not included in the article's Creative Commons license and your intended use is not permitted by statutory regulation or exceeds the permitted use, you will need to obtain permission directly from the copyright holder. To view a copy of this license, visit http://creativecommons.org/licenses/by/4.0/. 

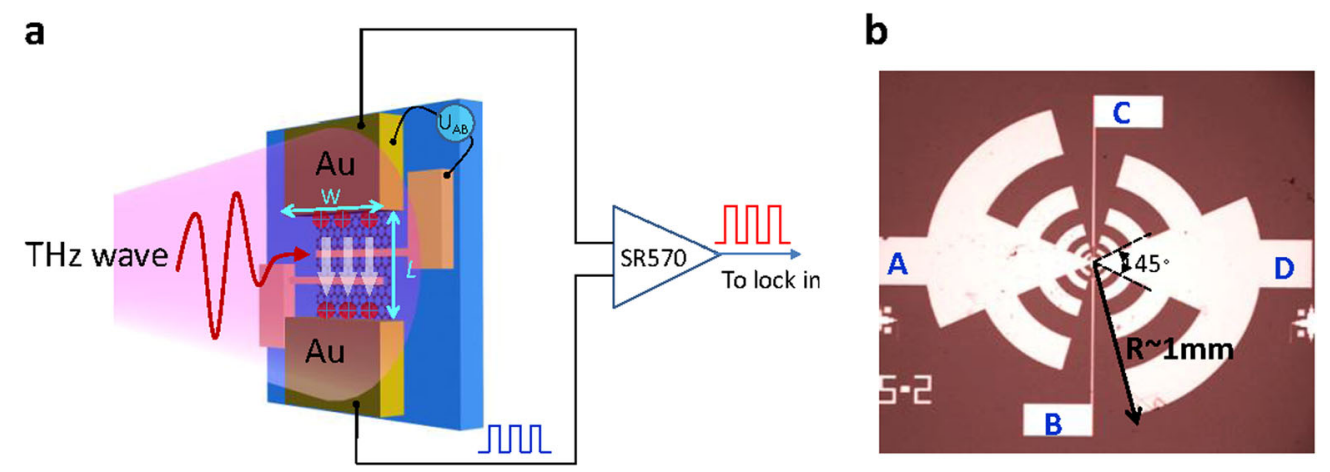

Fig. 1 a Schematic of a graphene-based THz detector under bias-field configuration: the graphene-channel is contacted by two log-antenna arms consisting of $\mathrm{Cr} / \mathrm{Au}$ stacks and two paired fingers in the middle, which results in a four-terminal electrical configuration marked $\mathrm{A}, \mathrm{B}, \mathrm{C}$, and $\mathrm{D}$ in the SEM image in $\mathbf{b}$. The channel features a length $L \sim 6 \mu \mathrm{m}$ and different widths $W$ of 60 and $6 \mu \mathrm{m}$ in the studied devices; the antenna arm features an outer radius of $R \sim 1 \mathrm{~mm}$ and a circular tooth span angle of $\pi / 4$. For more details about the device fabrication process, please see the Materials and Methods section

is usually quite low due to the weak light absorption of $2.3 \%$ through the interband transition as well as a large dark current ${ }^{15}$. Nevertheless, by utilizing the Drude response of free electrons in combination with heavy chemical doping, one can achieve $40 \%$ photon absorption in a single graphene layer in the mid-/far-infrared band of the electromagnetic spectrum ${ }^{16-19}$. Therefore, graphene is one of the most preferred materials for $\mathrm{THz}$ optoelectronics. Graphene-based $\mathrm{THz}$ detectors have been reported to exhibit suitable performance in the framework of plasma self-mixing and thermoelectric effects. The plasma self-mixing effect can be achieved via the asymmetrical antenna coupling of a $\mathrm{THz}$ field between a source/drain and gate, during which the nonlinear rectification of carrier transport leads to direct detection with a responsivity of approximately $0.04 \sim 20 \mathrm{~V} / \mathrm{W}^{19-22}$. Moreover, thermoelectric $\mathrm{THz}$ detection in graphene can be achieved by asymmetric contacting, which produces a Seebeck coefficient difference and direct photocurrent along the channel due to metal-induced asymmetrical doping $^{18}$, and the responsivity can reach as high as $15 \mathrm{~V} /$ W. While high-performance graphene-based devices have become increasingly critical to satisfying the requirements of everyday applications, the current implementation of direct $\mathrm{THz}$ detection is hindered by the lack of sufficient photoelectric gain ${ }^{23}$. The superior performance of graphene can be achieved by engineering the bias-field and electromagnetic size effects to manipulate the hot-carrier distribution. The results of the current study demonstrate the important role of carrier- $\mathrm{THz}$ field interactions in detection, which enable switching between the thermophotovoltaic (PV) and photoconductive (PC) modes, thereby producing previously unattainable levels of photoelectric gain.

\section{Materials and methods \\ Device fabrication}

The detector was fabricated using chemical vapor deposition (CVD)-grown single-layer graphene, which was transferred onto a highly resistive $\mathrm{Si} / \mathrm{SiO}_{2}(300 \mathrm{~nm})$ substrate for antenna patterning. The purpose of using a highly resistive Si substrate instead of a highly doped one was to eliminate the reflective loss of the incident wave, as the $\mathrm{THz}$ wavelength is at least two orders of magnitude longer than the dielectric layer thickness. The graphenechannel was contacted symmetrically by a log-periodic antenna to maximize the field confinement along the channel rather than perpendicular to it (see Fig. 1 and 2 (a), (b)). Thus, particular asymmetrical boundary conditions related to previously reported photothermoelectric $(\mathrm{PTE})^{18,19}$ and plasma-wave effects $^{21,22}$ were not considered. Oxygen-based plasma etching was performed after the antenna patterning and lift-off processes to form a graphene-channel (for more details, see Fig. S1, Supplementary Information). Additional paired contact fingers with different slit widths $s$ were then fabricated in the middle of channel by electron beam lithography, resulting in a four-terminal configuration ${ }^{24,25}$. The terminals are labeled A, B, C, and D in Fig. 1b. Thus, the device response depended specifically on the different electrical connections among these terminals. The homogeneity of the formed graphene-channel was confirmed by Raman scattering spectroscopy, and the current-voltage output characteristics with terminals $\mathrm{A}$ and $\mathrm{D}$ connected were determined for all the fabricated devices. It could also be inferred from the blueshift of the optical phonon-related $G$ peak in the Raman spectrum that the Fermi level relative to the Dirac point of the transferred sample is approximately $0.3 \mathrm{eV}$ (see Fig. S7, Supplementary Information). 


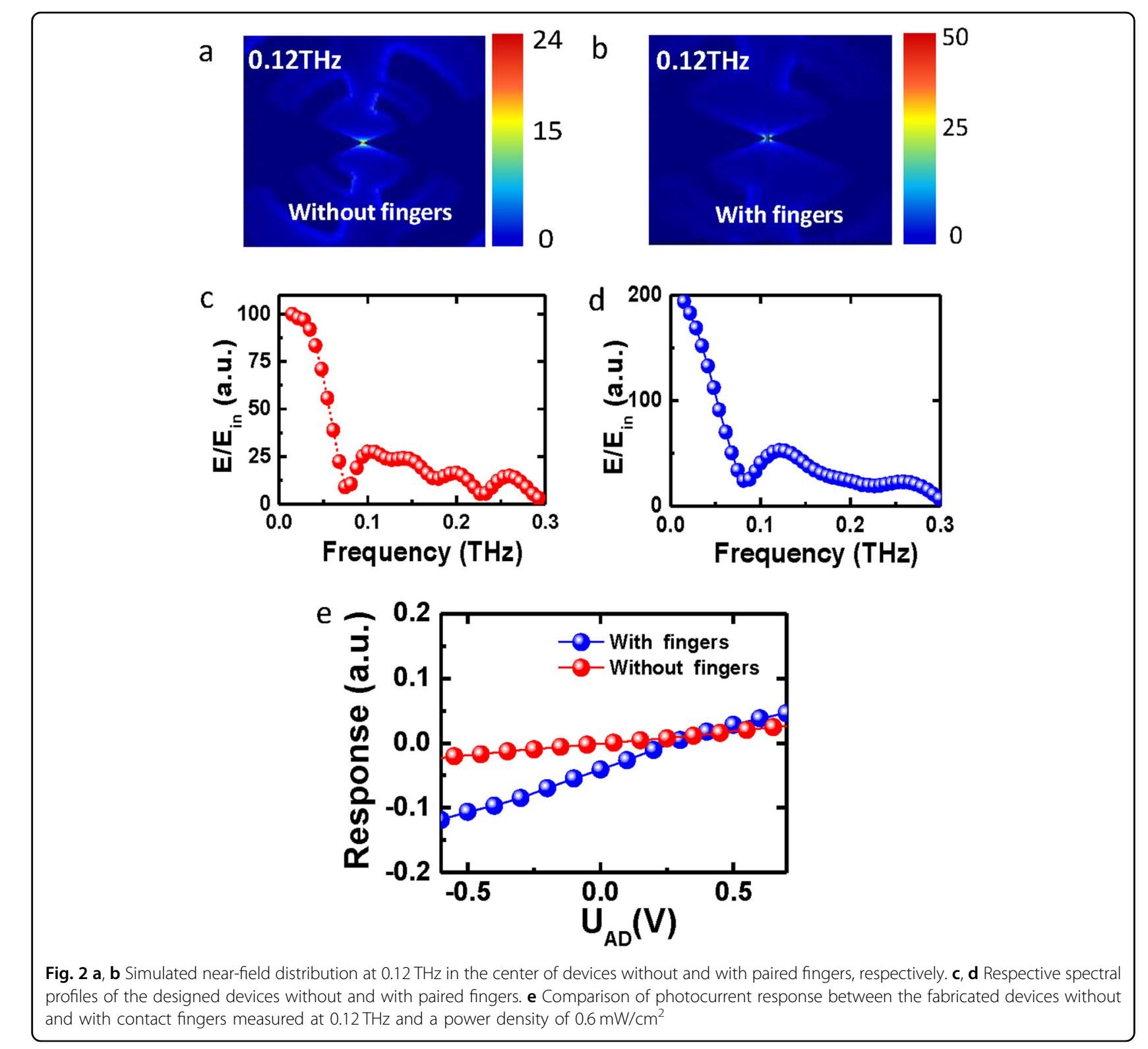

\section{Simulations}

Because the initial motivation of our work was to employ the electromagnetic size effect to enhance $\mathrm{THz}$ absorption, we performed a full-vector Finite Difference Time Domain simulation to better understand the electromagnetic size effect of the fabricated devices before further performance characterization. The geometrical parameters of the model strictly follow the structure of the fabricated devices. The metal layer was considered a perfect conductor, with perfectly matched layer (PML) absorbing boundary conditions around the device to remain consistent with the experimental conditions. Figure 2 shows the $\mathrm{THz}$ near-field distribution near the center of the designed devices. The electromagnetic size effect can be tailored in the center of the antenna by using paired contacts or by shortening the channel length/ antenna gap, both of which aim to enhance the dipole oscillation and capacitive coupling across the gap ${ }^{25}$. Figure 2(a), (b) show the corresponding amplitudes of the $\mathrm{THz}$ field at $0.12 \mathrm{THz}$ for the same device without and with the paired contacts. The comparison of these two configurations shows that the amplitude of the $\mathrm{THz}$ field for the latter configuration is much larger than that for the former one, which indicates that the additional paired contacts can significantly improve the electromagnetic coupling. The spectral characteristics of the devices without and with paired fingers are depicted in Fig. 2(c), (d), respectively, from which it can be determined that the localized near field in the center of the antenna is more than doubled with the incident frequencies being tuned 
from 0.04 to $0.2 \mathrm{THz}$. The largest field amplitude is observed at approximately $0.04 \mathrm{THz}(\lambda \sim 8 \mathrm{~mm})$ when the diameter of the antenna $(D \sim 2 \mathrm{~mm})$ is consistent with the wavelength $\lambda / n_{\text {sub }}$ of the electromagnetic wave in the substrate (the refractive index $n_{\text {sub }}$ is approximately 3.5 for highly resistive silicon). On the other hand, the incorporation of the paired fingers does not clearly alter the spectral profile of the antenna, and a broad photoresponse from 0.08 to $0.15 \mathrm{THz}$ is well preserved. The above-mentioned simulated results were confirmed experimentally with the fabricated device, as shown in Fig. 2e, which shows that the photocurrent responses at $0.12 \mathrm{THz}$ for the devices with and without paired contacts differ by a factor of four. Thus, our strategy of using paired contacts to enhance performance is valid.

\section{Results}

We characterized two types of devices with different resistances and structures. The $I-V$ characteristics of the fabricated devices were measured beforehand using an Agilent B2912A (see more details in Figs. S2 and S4, Supplementary Information). The electromagnetic response properties of the fabricated devices were characterized using a lock-in technique with a reference signal generated from an Agilent E8257D microwave source, which was subsequently connected to a VDI tripler to cover photon frequencies from 0.04 to $0.12 \mathrm{THz}$. The $\mathrm{THz}$ wave was outer-coupled with a horn antenna at a power density of $0.6 \mathrm{~mW} / \mathrm{cm}^{2}$. To study the detection mechanism and response speed of the devices, $0.12 \mathrm{THz}$ radiation was used under fast on/off modulations.

Figure 3a, $\mathrm{d}$ show 3D schematics of the wide- $(W \sim 60$ $\mu \mathrm{m}$, with $R_{\mathrm{AD}} \sim 100 \Omega$ ) and narrow- (W $6 \mu \mathrm{m}$, with $R_{\mathrm{AD}}$ $\sim 1 \mathrm{k} \Omega$ ) channel devices, respectively. Figure $3 \mathrm{~b}$, e show the measured photocurrent $I_{\mathrm{ph}-\mathrm{AD}}$ vs. the electrical bias $U_{\mathrm{AD}}$ for the corresponding devices. In addition, for the narrow-channel device shown in Fig. 3d, the slit width $s$ between the two fingers was varied from 0.5 to $1.5 \mu \mathrm{m}$ to further verify the electromagnetic size effect. The resistance $R_{\mathrm{AD}}$ remained nearly constant even as the slit was varied (see Fig. S8a, Supplementary Information). The photocurrent response in Fig. $3 \mathrm{~b}$ is only approximately two times higher than that in Fig. 3e, even though the resistance of the wide-channel device was an order of magnitude smaller than that of the narrow-channel one. Therefore, the voltage responsivity of the narrow-channel device should have been much higher, due to improved $\mathrm{THz}$ photon funneling in the narrow-channel device (more circular teeth can be achieved in a narrow-channel device, which is beneficial for $\mathrm{THz}$ field focusing; see the simulated results in Fig. S11a, b, Supplementary Information). Figure $3 \mathrm{~b}$, e show that non-zero crossings consistently occurred in the studied devices, particularly for those with a narrower channel (Fig. 3e). Such behavior is attributed to the non-equilibrium hot-carrier diffusion triggered by the asymmetrical $\mathrm{THz}$ near-field distribution along the channel due to the dislocation of paired fingers during fabrication (see Figs. 3g and Fig. S11c, Supplementary Information).

Formally, the thermoelectric effect can be given by ${ }^{26}$

$$
I_{\mathrm{ph}-\mathrm{AD}} \propto \int S(x) \frac{\mathrm{dT}_{\mathrm{h}}}{\mathrm{d} x} \mathrm{~d} x \text { or }\left(S_{1}(x)-S_{2}(x)\right) \nabla T_{h}(x)
$$

where $S=-\left.\frac{\pi^{2} \mathrm{k}_{\mathrm{B}}^{2} \mathrm{~T}}{3 e} \frac{1}{\sigma} \frac{\mathrm{d} \sigma}{\mathrm{d} E}\right|_{E=E_{\mathrm{F}}} \sim 15 \mu \mathrm{V} / \mathrm{K}$ is the Seebeck coefficient retrieved from the Mott relation, which depends on the doping level of graphene ${ }^{27,28}$, and $\mathrm{d} T_{\mathrm{h}} / \mathrm{d} x$ is the temperature gradient along the channel depending on local photon absorption. Equation 1 indicates that the thermoelectric current can be generated either by a temperature gradient within a non-zero Seebeck coefficient or by the local Seebeck coefficient difference. The doping distribution of the graphene-channel in our devices was homogeneous, with a Fermi-level difference less than $0.05 \mathrm{eV}$ (see Figs. S2 and S8 in Supplementary Information $)^{27}$; therefore, the difference was negligible for producing a sizable Seebeck coefficient difference. In this respect, thermoelectric photocurrent arises from the temperature gradient induced by the asymmetrical $\mathrm{THz}$ field coupling along the channel, rather than from differences in the Seebeck coefficient ${ }^{28}$. Because of carrier (hole) diffusion from the hot to the cool side, as shown in Fig. 3h, a net current flows along the channel in the absence of the electrical bias $U_{\mathrm{AD}}$. The temperature rise of carriers after local photon absorption can be estimated following the zero-bias photocurrent $I_{\mathrm{ph}-\mathrm{AD}}$ based on the relationship $\Delta T_{\mathrm{h}}=\Delta U_{\mathrm{ph}-\mathrm{AD}} / S=I_{\mathrm{ph}-\mathrm{AD}} R_{\mathrm{AD}} / S$, where $\Delta U_{\mathrm{ph}-\mathrm{AD}}$ is the open-circuit photovoltage. Therefore, the temperature rise $\Delta T_{\mathrm{h}}$ for hot carriers should be $<10$ and $60 \mathrm{~K}$ for the wide- and narrow-channel devices, respectively.

Furthermore, the photocurrent $I_{\text {ph-AD }}$ grows linearly by more than an order of magnitude under a finite electrical bias $U_{\mathrm{AD}}$. Because the PTE effect induces direct photocurrent generation and does not change with the static electric field, the increase in the photocurrent with the bias voltage can be attributed to the onset of additional resistance changes in the channel under $\mathrm{THz}$ radiation. Two competing potential photoresponse mechanisms require an electrical bias: (i) the bolometric effect, which is caused by changes in carrier mobility due to lattice heating, and (ii) the photoconductive effect, which is caused by changes in carrier number. Thus, the total dc current can be formulated as $I_{\mathrm{AD}}{ }^{(+)}=I_{\mathrm{AD} \text {-off }}{ }^{(+)}-\left|I_{\mathrm{B}}\right|+I_{\mathrm{PE}}$ $+I_{\mathrm{pc}}{ }^{(+)}$. The last term $I_{\mathrm{pc}} \sim e \mu n^{*} U_{\mathrm{AD}}$ is the photoconductive contribution due to the change in carrier density $n^{*}$ and $\left|I_{\mathrm{B}}\right|$ is the bolometric current summed with 
a

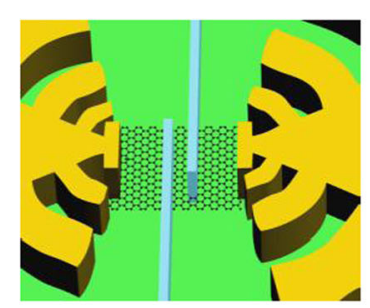

d

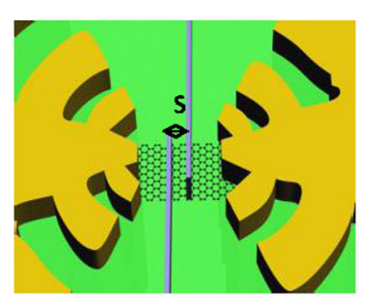

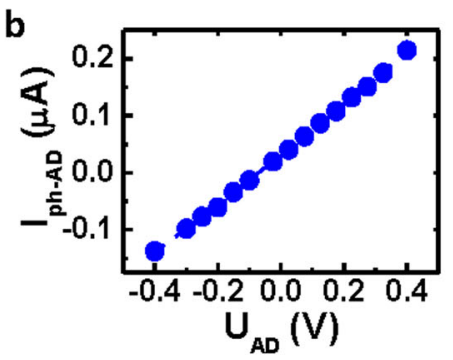

e
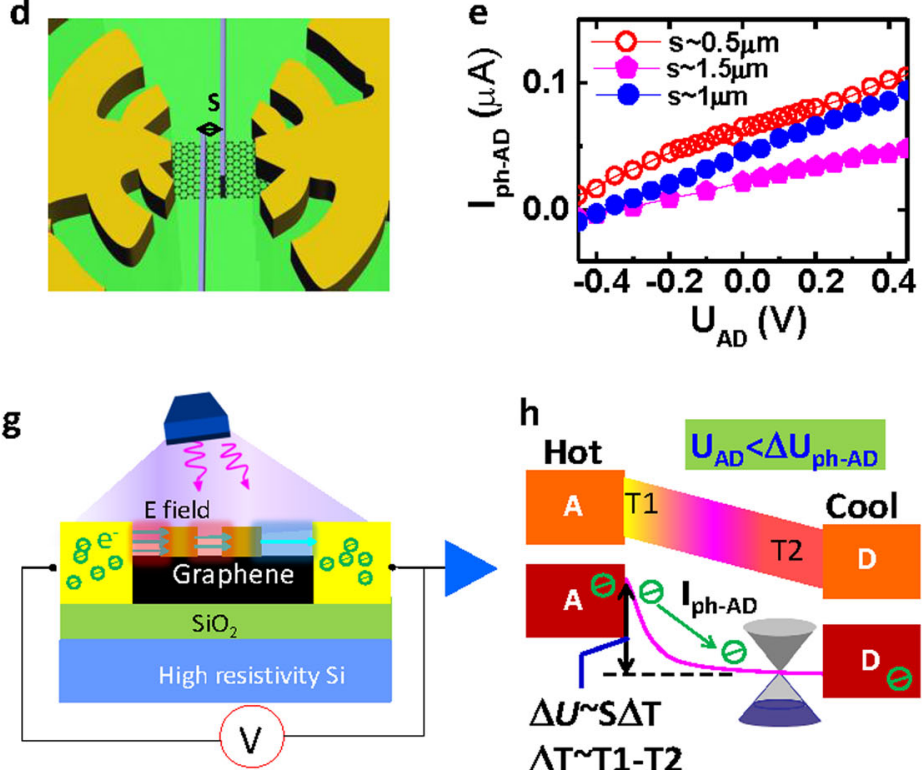

h

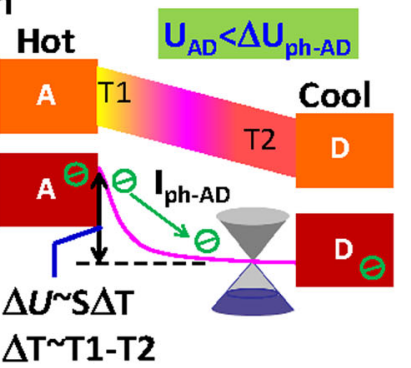

C

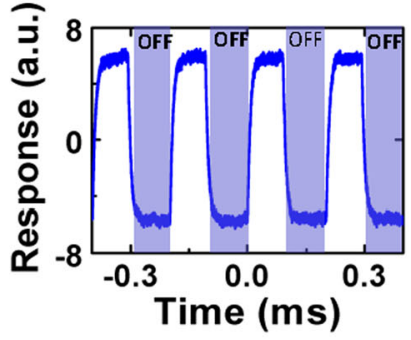

f

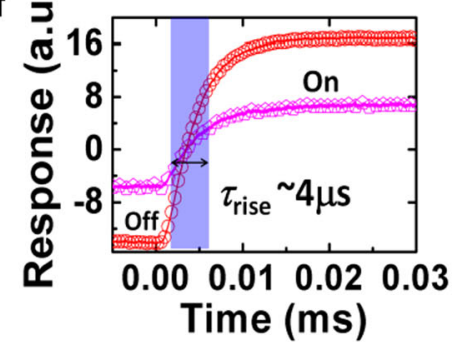

i

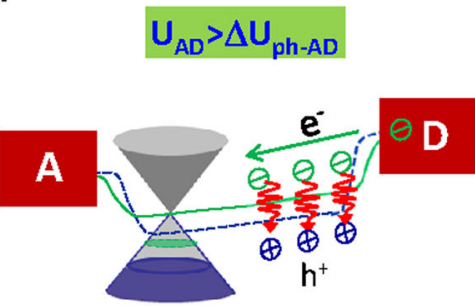

Fig. 3 The photoconductive response under $0.12 \mathrm{THz}$ radiation (power density of $0.6 \mathrm{~mW} / \mathrm{cm}^{2}$ ) for wide- and narrow-channel devices. a Schematic of the wide-channel device $(W \sim 60 \mu \mathrm{m})$. b Bias voltage $U_{\mathrm{AD}}$ dependence of the photocurrent $I_{\text {ph-AD. }}$. Temporal response of the wide-channel device under fast on/off THz radiation modulation. $\mathbf{d}$ Schematic of the narrow-channel device $(W \sim 6 \mu \mathrm{m})$, where $s$ denotes the slit width between the two paired contacts. e Bias voltage $U_{A D}$ dependence of photocurrent ph-AD $_{\text {in }}$ devices with different slits. $\mathbf{f}$ Response/recovery times for the narrowchannel device following modulated $\mathrm{THz}$ radiation. $\mathbf{g}$ Cross-section schematic of the electrical configuration for photoconductive measurement. The spaces between the contacts are shown in different colors representing the E-field distribution for the case in which the paired fingers are dislocated from the center. $\mathbf{h}$ Zero-bias photocurrent response due to hot-carrier diffusion from the hot to the cool side when $U_{A D}<\Delta U_{\text {ph-AD. }}$ i Photoconductive photocurrent response under finite bias voltage $U_{A D}>\Delta U_{\text {ph-AD; }}$ the generated hot carriers are efficiently extracted under finite bias with a photoconductive gain. $\Delta U_{\text {ph-AD }}=I_{\text {ph-AD }} \cdot R_{\mathrm{AD}}$ is the open-circuit voltage

the opposite sign due to the metallic behavior of graphene under lattice heating ${ }^{29}$.

To determine the dominant mechanism of linear photocurrent growth, the absorbed power must be accurately determined first ${ }^{30}$. The absorption rate of graphene in this work is mainly attributed to the Drude response and can be expressed analytically as $\eta=\frac{P_{\text {abs }}}{P_{0}}=\frac{\operatorname{Re}[\sigma(\omega)]\left|E_{\mathrm{t}}\right|^{2}}{E_{0}^{2} / Z_{0}}=\frac{4 Z_{0} \operatorname{Re}[\sigma(\omega)]}{\left|1+n+Z_{0} \sigma(\omega)\right|^{2}} \approx 13 \%\left(\right.$ where $\quad n_{\text {sub }} \sim 3$ is the $\mathrm{Si}$ index and $Z_{0}=377 \Omega$ is the impedance of free space), following the methodology of Ref. ${ }^{31}$. Because the incident power at $0.12 \mathrm{THz}$ onto the active channel is approximately $0.4 \mu \mathrm{W}$, based on the electromagnetic simulation (see Fig. S10, Supplementary Information), the absorbed power $P_{\text {abs }}$ can be determined to be approximately $0.05 \mu \mathrm{W}$. The thermal resistance of graphene is proved to be on the order of $(T / 5)^{-3} \mathrm{~K} / \mathrm{nW} \sim$ $4.6 \times 10^{3} \mathrm{~K} / \mathrm{W}(T \sim 300 \mathrm{~K} \text { is room temperature })^{32}$, and thus, the temperature rise $\Delta T_{\mathrm{L}}$ is on the order of $10^{-4}$ and $10^{-5} \mathrm{~K}$, corresponding to the incident and absorbed power, respectively. The temperature coefficient of resistance in our device was approximately $4 \times 10^{-3} \% / \mathrm{K}$; thus, the resistance change should be $<0.004$ and $0.04 \Omega$ for the wide- and narrow-channel devices, even if we suppose the temperature rise $\Delta T_{\mathrm{L}}$ is approximately $1 \mathrm{~K}$. On the other hand, based on the experimental results shown in Fig. 3b, $\mathrm{e}$, the resistance change can be determined to be approximately 0.1 and $5 \Omega$ for the wide- and narrowchannel devices respectively, based on the relationship $\Delta R_{\mathrm{AD}} \sim I_{\mathrm{ph}-\mathrm{AD}} R_{\mathrm{AD}}^{2} / U_{\mathrm{AD}}$. Such a large discrepancy 
between the experimental and calculated results implies that the bolometric effect can be ruled out, and the resistance change under $\mathrm{THz}$ radiation is attributed to the change in carrier number. Although the photocarriers cannot be excited directly via interband transition, they can be generated by the charge transfer driven by the thermoelectric potential, as shown Fig. 3i. Under electrical bias $U_{\mathrm{AD}}$, the thermoelectric photocurrent can be neglected, and photoconduction becomes the dominant effect when $U_{\mathrm{AD}}>\Delta U_{\mathrm{ph}-\mathrm{AD}}\left(\Delta U_{\mathrm{ph}-\mathrm{AD}}\right.$ is the open-circuit photovoltage), as shown in Fig. 3i.

To characterize the response speed, the devices were subjected to $\mathrm{THz}$ radiation with fast on/off modulation, as shown in Fig. 3c, f. The pulsed shape was well preserved with a good signal-to-noise ratio for both the wide- and narrow-channel devices. The response time, defined as the timescale between 10 and $90 \%$ on the rising edge or, analogously, on the falling edge, was approximately $4 \mu \mathrm{s}$ (see Fig. 3f and Fig. S6 in Supplementary Information). This response speed is already adequate for video-rate $\mathrm{THz}$ imaging applications and is actually limited by the transmission loss of current readout electronic systems.

The results in Fig. 4 are based on the electrical contacting configuration shown in Fig. 4a. The following analysis and discussion focus on the devices with a narrow-channel due to their higher performance. The output photocurrent $I_{\text {ph-AD }}$ was measured with application of a finite bias voltage $U_{\mathrm{AB}}$ between contacts $\mathrm{A}$ and $\mathrm{B}$. Figure $4 \mathrm{~b}$ shows that $I_{\mathrm{AD}}$ can be pinched off and its sign reversed when $U_{\mathrm{AB}}$ is sufficiently large. During this process, $I_{\mathrm{AD}}$ shows a nonlinear relationship with the bias voltage $U_{\mathrm{AB}}$. Owing to an electrical connection similar to that of a triode, the current will change significantly depending on the sign of the bias voltage. Therefore, we referred to the process as a "bias-field effect", as no strong PN junction effects such as those in a triode can arise in the device due to the absence of a bandgap. However, such nonlinear behavior is favorable to the rectification of $\mathrm{THz}$ signals (see, for example, the self-mixing in Fig. S3, Supplementary Information) depending on the incident frequency and metal-graphene interface. To clarify the $\mathrm{THz}$ detection mechanism with this configuration, here we consider a more general case with the detuned frequency far from the self-mixing regime. Figure $4 \mathrm{~d}$ displays the measured signal vs. the $U_{\mathrm{AB}}$ bias voltage, where the photocurrent $I_{\mathrm{ph}-\mathrm{AD}}$ experiences its minimum at $U_{\mathrm{AB}} \sim$ $0.5 \mathrm{~V}$ and then increases almost linearly as $U_{\mathrm{AB}}$ continues to increase. During this process, no additional bias voltage $U_{\mathrm{AD}}$, such as that in Fig. 3, is needed, as shown in Fig. 4d, which indicates the existence of a direct photocurrent generation mechanism.

Generally, there are also two competing mechanisms for direct photocurrent generation: (i) the above-mentioned thermoelectric effect and (ii) the plasma-wave self-mixing effect, which is related to the rectification of the nonlinear $I V$ characteristics. The photocurrent associated with the self-mixing effect is proportional to the first derivative of conductance $^{22}$ :

$$
I_{\mathrm{ph}-\mathrm{AD}} \propto \frac{1}{\sigma_{\mathrm{AD}}} \times \frac{\mathrm{d} \sigma_{\mathrm{AD}}}{\mathrm{dU} \mathrm{AB}_{\mathrm{AB}}}
$$

To generate photocurrent, both competing mechanisms require asymmetrical boundary conditions. In previously reported graphene $\mathrm{FETs}^{22}$, the self-mixing effect can arise due to asymmetrical antenna coupling between the source and gate, leading to a longitudinal direct electric field and photocurrent/photovoltage generation. The photocurrent derived from the self-mixing effect reverses its sign following the derivative $\mathrm{d} \sigma_{\mathrm{AD}} / \mathrm{d} U_{\mathrm{AB}}$ near the Dirac point. Figure $4 \mathrm{c}$ depicts the derivative of the conductance $\sigma_{\mathrm{AD}} /$ $\mathrm{d} U_{\mathrm{AB}}$ as a function of $U_{\mathrm{AB}}$. The derivative changes sign found at $U_{\mathrm{AB}} \sim 0.5 \mathrm{~V}$ in Fig. 4c. However, the linear shape of the photocurrent curve in Fig. 4d departs significantly from that in Fig. 4c, and there are no hints of sign change in the photocurrent at any bias points. To this case, the plasma self-mixing effect should be ruled out. Further proof of the role played by the self-mixing effect in photocurrent generation can be found in the Supplementary Information, with paired contact fingers replaced by other types of metallic contacts (e.g., Ti/Au, see Fig. S2, Supplementary Information), where the critical point of $U_{\mathrm{AB}}$ for photocurrent polarity inversion coincides with the sign change of the derivative $\mathrm{d} \sigma_{\mathrm{AD}} / \mathrm{d} U_{\mathrm{AB}}$. These results clearly demonstrate that the tuning conditions required by the self-mixing effect depend on the properties of metal-graphene contacts, such as parasitic capacitance and resistance. Furthermore, the results indicate an alternative route to achieving nonlinear rectification of $\mathrm{THz}$ waves via bias-field configuration. In the absence of self-mixing, the hot carriers still prevail due to the Drude response, and the thermoelectric response can generally be activated. Figure $3 \mathrm{e}$ illustrates the physics underlying the photocurrent response: (i) the asymmetrical boundary is satisfied when the bias voltage $U_{\mathrm{AB}}$ is applied because of charge transfer at the metal-graphene interface (Fig. 3e) ${ }^{33,34}$. (ii) Diffusion of non-equilibrium hot carriers under $\mathrm{THz}$ excitation between contacts $\mathrm{A}$ and $\mathrm{D}$ cannot be neglected due to the non-symmetrical carrier distribution (Fig. 3f, i.e., $\int_{\mathrm{AD}} S(x) \mathrm{d} T_{\mathrm{h}} \neq 0$ ), which would lead to direct photocurrent generation. As the bias voltage $U_{\mathrm{AB}}$ increases, the Fermi level of graphene continues to shift upward, and the Seebeck coefficient difference among the local channels also increases, facilitating thermoelectric photocurrent generation, as shown in Fig. 4d. Following this process, the photocurrent is improved by more than an order of magnitude when $U_{\mathrm{AB}}$ is only $1 \mathrm{~V}$. Figure $4 \mathrm{~d}$ shows that the bias-field-induced photocurrent is at least eight times higher than that generated through the 

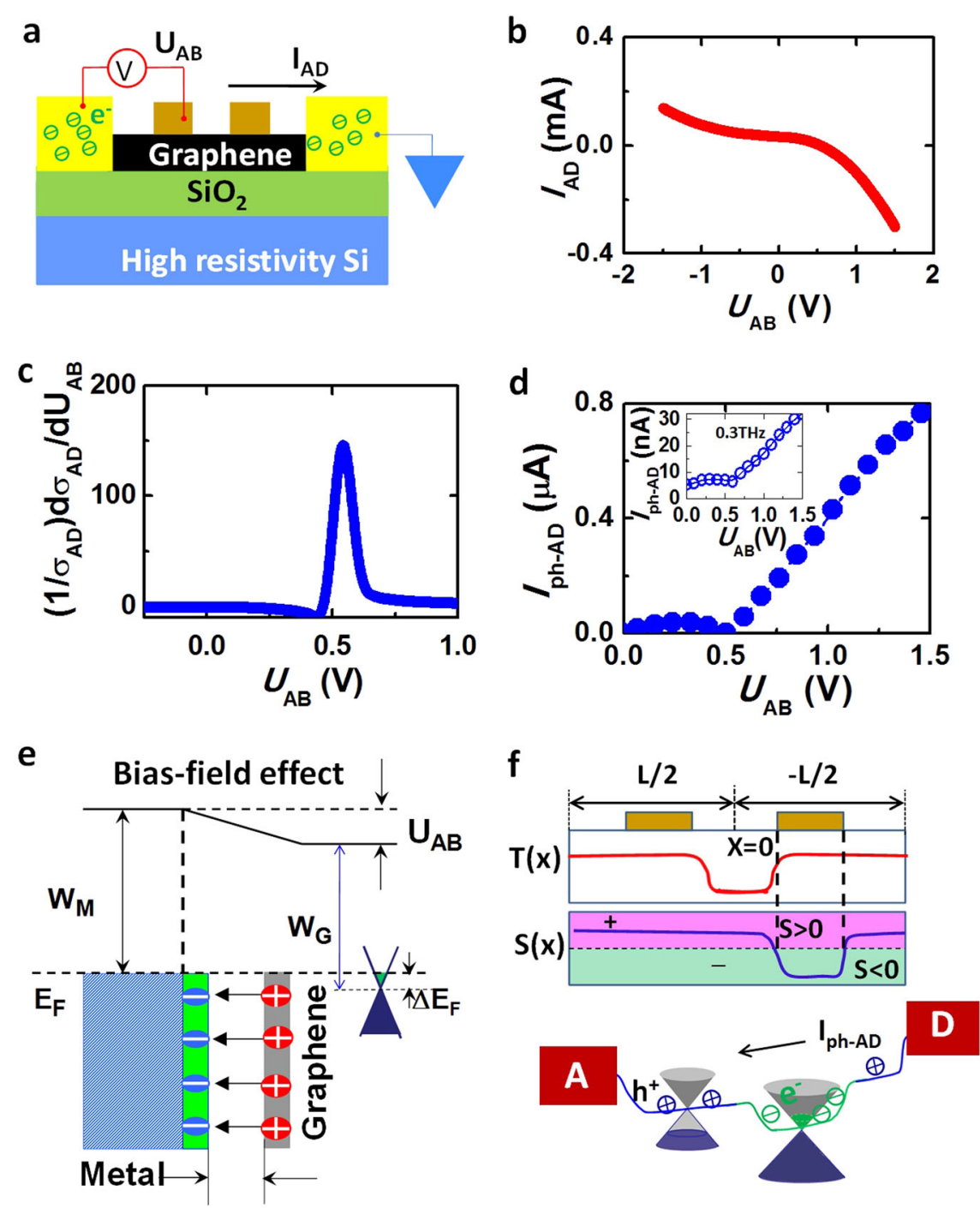

Fig. 4 The direct photocurrent/photovoltaic response $I_{\text {ph-AD }}$ under $0.12 \mathrm{THz}$ radiation (power density of $0.6 \mathrm{~mW} / \mathrm{cm}^{2}$ ), tuned by the bias voltage $U_{A B}$ between contacts $\mathrm{A}$ and $\mathrm{B}$. $\mathbf{a}$ Schematic of the bias-field configuration. The photocurrent $I_{\mathrm{ph}-\mathrm{AD}}$ is readout as a function of the bias voltage $U_{\mathrm{AB}}$. $\mathbf{b} U_{\mathrm{AB}}$ dependence of the dark current $I_{A D}$; the bias voltage $U_{A D}$ is approximately $100 \mathrm{mV}$. c Derivation of conductance $\sigma_{A D} v s$. $U_{A B}: \sigma_{A D}{ }^{-1} \mathrm{~d} \sigma_{A D} / d U_{A B}$. $\mathbf{d}$ Direct photocurrent $I_{\text {ph-AD }}$ VS. $U_{A B}$ under $0.12 \mathrm{THz}$ radiation. The inset shows a similar trend of photocurrent response at $0.3 \mathrm{THz}$. e Band diagram of the "bias-field effect" induced by direct photocurrent generation. $\mathbf{f}$ The symmetry of carrier distribution is broken when the electrical bias $U_{A B}$ is applied due to charge transfer between the metal and graphene ${ }^{33,34}$, which ultimately facilitates non-equilibrium hot-carrier diffusion in our devices. $T(x)$ denotes the hot-carrier temperature distribution; $S(x)$ is the Seebeck coefficient

photoconductive effect (Fig. 3e). A similar effect can be observed under $\mathrm{THz}$ radiation at other frequencies, e.g., $0.3 \mathrm{THz}\left(1 \mathrm{~mW} / \mathrm{cm}^{2}\right)$, as shown in the inset of Fig. $4 \mathrm{~d}$, and at a lower frequency of $0.04 \mathrm{THz}$ (see Figs. S2 and S5 in Supplementary Information), all of which exhibit similarly drastic enhancements in direct photocurrent under higher bias fields. In this study, the photocurrent of $30 \mathrm{nA}$ observed at $0.3 \mathrm{THz}$ was an order of magnitude weaker than that recorded at $0.12 \mathrm{THz}$, likely due to the decreased coupling efficiency of electromagnetic waves according to the simulated results shown in Fig. 2c, d.
To evaluate device sensitivity, the voltage/current responsivity and noise-equivalent power (NEP) of the detector can be obtained via the following equation:

$$
R_{\mathrm{v}}=\frac{I_{\mathrm{ph}-\mathrm{AD}} R_{\mathrm{AD}}}{P_{\mathrm{in}}}, \mathrm{NEP}=\frac{\mathrm{V}_{\mathrm{N}}}{R_{\mathrm{V}}}
$$

where $R_{\mathrm{AD}}$ is the resistance and $P_{\mathrm{in}} \sim P_{\text {out }} S_{\mathrm{A}} / S_{\mathrm{b}}$ is the incident power on the device; here, $S_{\mathrm{A}}\left(\sim 3 \mathrm{~mm}^{2}\right)$ is the total area of the device, including the antenna area that overlaps with the plane-wave output in the beam spot area $S_{\mathrm{b}}\left(S_{\mathrm{b}} \sim 10 \mathrm{~cm}^{2}\right)$ and $P_{\text {out }}$ is the output power $(6 \mathrm{~mW}$ at $0.12 \mathrm{THz}$ ). Figure $5 \mathrm{a}$ shows the $U_{\mathrm{AD}}$ bias dependence of 

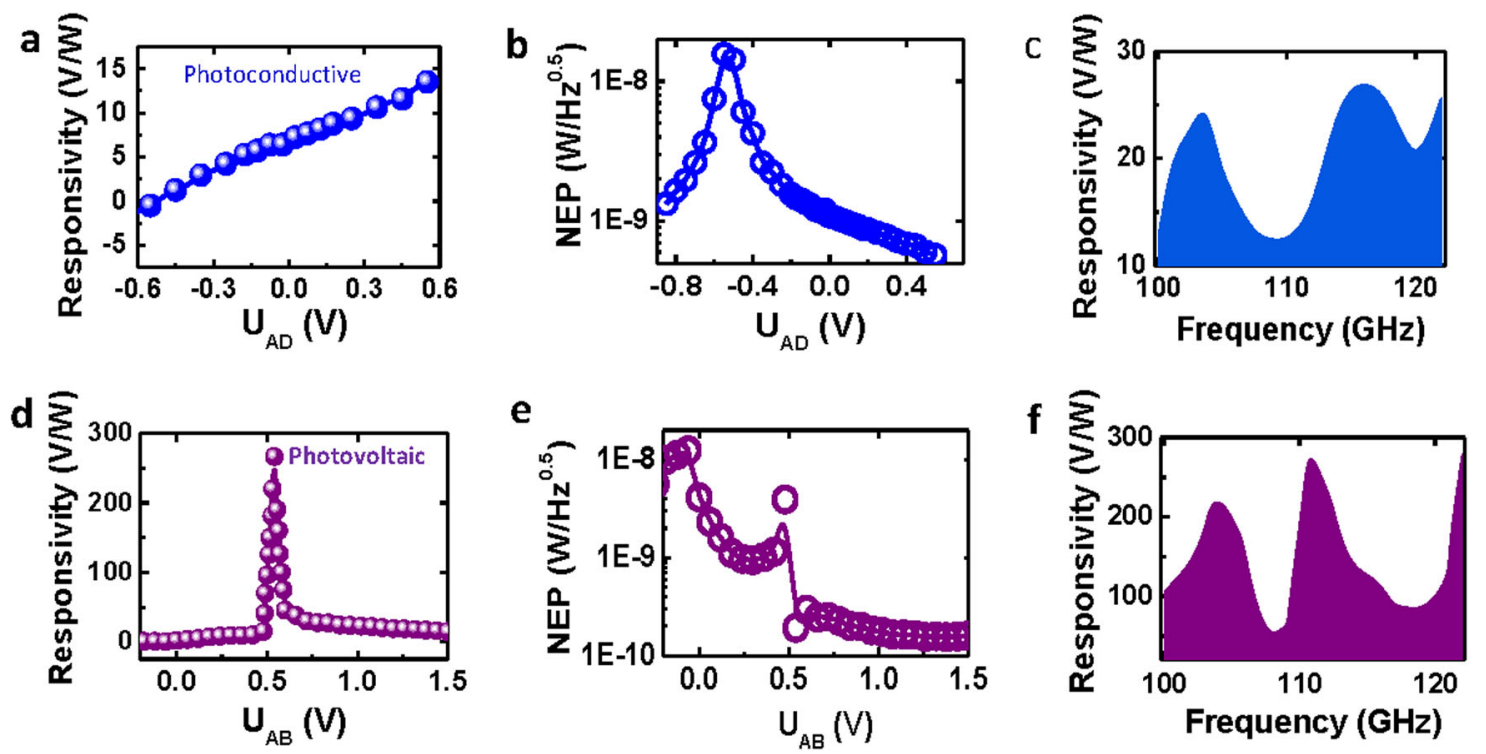

Fig. $\mathbf{5}$ a-c Photoconductive mode. a Voltage responsivity of the device as a function of bias voltage $U_{A D}$ operating in the photoconductive mode at $0.12 \mathrm{THz}$. b Johnson-noise limited NEP vs. electrical bias $U_{A D}$. c Response spectrum of the photoconductive mode, where $U_{A D}$ is $0.6 \mathrm{~V}$. $\mathbf{d}-\mathbf{f}$ Photovoltaic/direct photocurrent mode with bias-field configuration: $\mathbf{d}$ Voltage responsivity as a function of electrical bias voltage $U_{\mathrm{AB}}$. $\mathbf{e}$ Corresponding NEP in the Johnson-noise limit. $\mathbf{f}$ Response spectrum of the photovoltaic/direct photocurrent mode, where $U_{A B}$ is $0.5 \mathrm{~V}$

the photoconductive responsivity for the narrow-channel devices. Although much lower photocurrent occurred in the narrow-channel devices than in the wider-channel devices, the voltage responsivity was improved by at least a factor of five, reaching a value of $15 \mathrm{~V} / \mathrm{W}$, and higher responsivity could be anticipated at a higher bias voltage $U_{\mathrm{AD}}$. Because the antenna size was the same for both the wide- and narrow-channel devices, the higher responsivity can be attributed to the tailorable near field in close proximity to the channel. In this regard, it is clear that the effective area for funneling the electromagnetic wave into the channel can be much smaller than the actual antenna area used in this work (see Figs. S9 and S10 in Supplementary Information). Therefore, the responsivity can be improved greatly by exploring the electromagnetic size effect of a subwavelength antenna with a smaller gap between the antenna arms to improve the dipole oscillation across the channel.

Based on the above-mentioned results, the maximum voltage responsivity of our device reached as high as 280 $\mathrm{V} / \mathrm{W}$, by employing the "bias-field effect" electrical configuration shown in Fig. 5d. Such remarkable enhancement in device performance is similar to that achieved by the plasma self-mixing effect, which produces a responsivity maximum near the threshold (see Supporting Information, Fig. S3) and can be understood as an interior loading effect $\left(\Delta U \sim R_{\mathrm{ch}} S \Delta T / R_{1}\right.$, where $R_{\mathrm{ch}}$ is the total resistance and $R_{1}$ is the hot spot channel resistance for thermoelectric generation) $)^{35}$. A similar voltage dependence could be observed at $0.3 \mathrm{THz}$, which showed the lowest efficiency due to the mismatched $\mathrm{THz}$ coupling with the current antenna structure, as shown in Figs. 2 and 4 . Nevertheless, the responsivity observed at $0.3 \mathrm{THz}$ was modest and could exceed $10 \mathrm{~V} / \mathrm{W}$, indicating performance comparable to that of other optimized detectors operating at a similar frequency ${ }^{36}$.

Another important parameter for representing the sensitivity of detectors is the NEP, which is extracted from the ratio $V_{\mathrm{N}} / R_{\mathrm{v}}$ by assuming that the main contribution to the noise is the thermal Johnson-Nyquist noise $\left(N_{\text {th }}\right)$ associated with the non-zero resistance of the FET channel $\left(V_{\mathrm{N}}=\sqrt{4 k_{\mathrm{B}} \mathrm{Tr}}\right)^{37}$, where $k_{\mathrm{B}}$ is Boltzmann's constant and $T$ is room temperature. Although this hypothesis neglects shot noise, it provides a lower limit for the NEP. Figure 5b, e show the NEP of our devices operating in the photoconductive and photovoltaic modes, respectively. In addition, the minimal $\mathrm{NEP}$ of our devices was $0.1 \mathrm{nW} / \mathrm{Hz}^{0.5}$ when operating in photovoltaic mode. For the photoconductive mode, the NEP was approximately five times larger $(\sim 0.5 \mathrm{nW} /$ $\mathrm{Hz}^{0.5}$ ), which is also moderate compared with recently reported results ${ }^{18,20}$. Therefore, switching between different working modes can be achieved within a single device, depending on the configuration of the electrical contacts and the subsequent readout set-up. Figure $5 \mathrm{c}, \mathrm{f}$ show the response spectra of the devices operating in different modes. The average responsivities of the devices operating in the photovoltaic $\left(U_{\mathrm{AB}} \sim 0.5 \mathrm{~V}\right)$ and photoconductive mode $\left(U_{\mathrm{AD}} \sim 0.6 \mathrm{~V}\right)$ were 100 and 10 $\mathrm{V} / \mathrm{W}$, respectively ${ }^{38}$. 


\section{Discussion}

Based on the foregoing discussion, it can be concluded that the considerable improvement in the NEP is ultimately the result of the enhanced responsivity originating from careful antenna design, interface control, and $\mathrm{THz}$ field focusing. The generation of PTE current can be formally boosted by either asymmetrical electromagnetic heating or dissimilar electric contacts with significant Seebeck coefficient differences. The main limitation of the PTE effect is the lack of sufficient internal gain, as can be obtained in a normal interband photon detector in which multiple charge carriers can be generated with only one absorbed photon ${ }^{35}$. Such difficulties can now be circumvented in a photoconductive mode with weak electrical or structural asymmetry. The hot carriers diffused from the metal-graphene interface are efficiently extracted under DC electric field, leading to a large photoconductive gain due to both the high carrier mobility and Fermi velocity of graphene. Furthermore, the bias-field-dependent effect clearly modifies the current-voltage characteristics or hot-carrier distribution, enabling a drastic enhancement in thermoelectric efficiency for direct photocurrent generation.

As previously mentioned, the responsivity observed for the devices fabricated in this work are still rather conservative estimations owing to the over-sized antenna and rather long channel length employed in the current design. On one hand, the device response mainly relies on the power density focused onto the channel rather than the antenna area. In our case, the antenna was an order of magnitude larger than previously reported ones ${ }^{16,18-20}$. Indeed, the antenna can retain a superior radiation funneling capability with a much smaller effective antenna area, even though it is smaller than the diffraction limit area defined by $S \sim\left(\lambda / 2 n_{\text {sub }}\right)^{2}\left(\lambda\right.$ is the wavelength and $n_{\text {sub }}$ $\sim 3.5$ is the refractive index of the substrate; also see the Supporting Information for a detailed calculation of the effective antenna area). On the other hand, the response also depends on the near-field tailoring of the received radiation from the antenna to the active channel. Thus, the performance can be improved by further exploring the electromagnetic size effect by shortening the channel length or enhancing the dipole interaction between the two opposite antenna arms, which is exactly what we proposed. Therefore, it is reasonable to expect that a re-designed antenna with a scaled area in conjunction with a distinct size effect can substantially boost the interaction between graphene and $\mathrm{THz}$ photons, which is essential to obtaining both higher responsivity and higher chip-level pixel intensity for potential $\mathrm{THz}$ imaging devices.

\section{Conclusions}

In summary, we demonstrated enhanced roomtemperature $\mathrm{THz}$ detection using antenna-coupled graphene transistors via the introduction of paired finger contacts, which enable different detecting modes driven by different mechanisms, including photoconductive, thermophotovoltaic and plasma self-mixing effects. In particular, through the manipulation of the electrical connection, a novel "bias-field effect" could be activated, leading to a drastic enhancement in $\mathrm{THz}$ detection ability with a responsivity of up to $280 \mathrm{~V} / \mathrm{W}$ and a Johnson-noise limited minimum noise-equivalent power (NEP) of $100 \mathrm{pW} / \mathrm{Hz}^{0.5}$ at room temperature, indicating a performance level competitive with that of the best mechanically exfoliated graphene thermoelectric detectors. In addition to well-established strategies, our work shows that the metal-graphene interface, workfunction engineering, and localized electromagnetic engineering can play prominent roles in improving $\mathrm{THz}$ detection. The room-temperature operation, fast response, high sensitivity, and low NEP of the devices reported in this work offer a promising alternative route to scalable, wafer-level production of high-performance graphene detectors.

\section{Acknowledgements \\ This work is supported in part by the State Key Program for Basic Research of China (2017YFA0205801, 2017YFA0305500, 2013CB632705), the National Natural Science Foundation of China (11334008, 61290301, 61521005, 61405230, 61675222), the Youth Innovation Promotion Association (CAS), Aviation Science Fund No. 20162490001. \\ Author details \\ 'State Key Laboratory of Infrared Physics, Shanghai Institute of Technical Physics, Chinese Academy of Sciences, 500 Yutian Road, Shanghai 200083, China. 'University of Chinese Academy of Sciences, No 19 A Yuquan Road, Beijing 100049, China. ${ }^{3}$ State Key Laboratory of Molecular Engineering of Polymers, Department of Macromolecular Science, Fudan University, Shanghai 200433, China. ${ }^{4}$ Hubei Collaborative Innovation Center for Advanced Organic Chemical Materials, Key Laboratory for the Green Preparation and Application of Functional Materials, Ministry of Education, School of Materials Science and Engineering, Hubei University, Wuhan 430062, China. ${ }^{5}$ Synergetic Innovation Center of Quantum Information \& Quantum Physics, University of Science and Technology of China, Hefei, Anhui 230026, China}

\section{Authors' contributions}

L.W., G.C., and X.S.C. conceived the project. L.W. designed the experiments. C.L. L., W.W.T., and L.D. conducted all the experiments. D.C.W., G.C., and J.H.L. participated in writing the manuscript. L.W., X.S.C., W.L., and G.C. analyzed the data. All authors contributed to the organization of the manuscript.

Conflict of interest

The authors declare that they have no conflict of interest.

Publisher's note

Springer Nature remains neutral with regard to jurisdictional claims in published maps and institutional affiliations.

Supplementary information is available for this paper at https://doi.org/ 10.1038/s41427-018-0032-7.

Received: 21 October 2017 Revised: 28 December 2017 Accepted: 21 January 2018.

Published online: 18 April 2018 


\section{References}

1. Ju, L. et al. Topological valley transport at bilayer graphene domain walls. Nature 520, 650-655 (2015).

2. Sui, M. et al. Gate-tunable topological valley transport in bilayer graphene. Nat Phys. 11, 1027-1031 (2015)

3. Giorgianni, F. et al. Strong nonlinear terahertz response induced by Dirac surface states in $\mathrm{Bi}_{2} \mathrm{Se}_{3}$ topological insulator. Nat. Commun. 7, 11421 (2016).

4. Mak, K. F., McGill, K. L., Park, J. \& McEuen, P. L. The valley Hall effect in MoS transistors. Science 344, 1489-1492 (2014).

5. Chen, S., Zheng, C., Fuhrer, M. S. \& Yan, J. Helicity-resolved Raman scattering of $\mathrm{MoS}_{2}, \mathrm{MoSe}_{2}, \mathrm{WS}_{2}$, and WSe, atomic layers. Nano Lett. 15, 2526-2532 (2015).

6. Lu, J. M. et al. Evidence for two-dimensional Ising superconductivity in gated MoS. Science 350, 1353-1357 (2015)

7. Navarro-Moratalla, E. et al. Enhanced superconductivity in atomically thin $\mathrm{TaS}_{2}$ Nat. Commun. 7, 11043 (2016)

8. Koppens, F. H. L. et al. Photodetectors based on graphene, other twodimensional materials and hybrid systems. Nat. Nanotechnol. 9, 780-793 (2014).

9. Grigorenko, A. N., Polini, M. \& Novoselov, K. S. Grapheneplasmonics. Nat. Photonics 6, 749-758 (2012).

10. Lundeberg, M. B. et al. Tuning quantum nonlocal effects in grapheneplasmonics. Science 357, 187-191 (2017).

11. Basov, D. N., Fogler, M. M. \& García de Abajo, F. J. Polaritons in van der Waals materials. Science 354, 195 (2016).

12. Choi, W. et al. High-detectivity multilayer $\mathrm{MoS}_{2}$ phototransistors with spectral response from ultraviolet to infrared. Adv. Mat. 24, 5832-5837 (2012).

13. Jariwala, D. et al. Hybrid, gate-tunable, van der Waals $\mathrm{p}$-n heterojunctions from pentacene and MoS. Nano Lett. 16, 497-503 (2016).

14. Buscema, M. et al. Photocurrent generation with two-dimensional van der Waals semiconductors. Chem. Soc. Rev. 44, 3691-3718 (2015).

15. Freitag, M., Low, T., Xia, F. \& Avouris, P. Photoconductivity of biased graphene Nat. Photonics 7, 53-59 (2013).

16. Yao, Y. et al. High-responsivity mid-infrared graphene detectors with antennaenhanced photocarrier generation and collection. Nano Lett. 14, 3749-3754 (2014).

17. Yao, Y. et al. Wide wavelength tuning of optical antennas on graphene with nanosecond response time. Nano Lett. 14, 214-219 (2014).

18. Tong, J., Muthee, M., Chen, S., Yngvesson, S. K. \& Yan, J. Antenna enhanced graphene THz emitter and detector. Nano Lett. 15, 5295-5301 (2015).

19. Ghahari, F. et al. Enhanced thermoelectric power in graphene: violation of the Mott relation by inelastic scattering. Phys. Rev. Lett. 116, 136802 (2016).

20. Viti, L. et al. Heterostructured hBN-BP-hBN nanodetectors at terahertz frequencies. Adv. Mat. 28, 7390-7396 (2016).
21. Viti, L. et al. Black phosphorus terahertz photodetectors. Adv. Mat. 27 5567-5572 (2015)

22. Vicarelli, L. et al. Graphene field-effect transistors as room-temperature terahertz detectors. Nat. Mater. 11, 865-871 (2012).

23. Otsuji, T. \& Shur, M. S. Terahertz plasmonics: Good results and great expectations. IEEE Microw. Mag. 15, 43-50 (2014).

24. Tomadin, A., Tredicucci, A., Pellegrini, V., Vitiello, M. S. \& Polini, M. Photocurrentbased detection of terahertz radiation in graphene. Appl. Phys. Lett. 103, 211120 (2013).

25. Seo, M. A. et al. Terahertz field enhancement by a metallic nano slit operating beyond the skin-depth limit. Nat. Photonics 3, 152-156 (2009).

26. Viti, L. et al. Efficient terahertz detection in black-phosphorus nano-transistors with selective and controllable plasma-wave, bolometric and thermoelectric response. Sci. Rep. 6, 20474 (2016).

27. Ghahari, F. et al. Enhanced thermoelectric power in graphene: violation of the Mott relation by inelastic scattering. Phys. Rev. Lett. 116, 136802 (2016).

28. Song, J. C., Rudner, M. S., Marcus, C. M. \& Levitov, L. S. Hot carrier transport and photocurrent response in graphene. Nano Lett. 11, 4688-4692 (2011).

29. Yin, Y., Cheng, Z., Wang, L., Jin, K. \& Wang, W. Graphene, a material for high temperature devices-intrinsic carrier density, carrier drift velocity, and lattice energy. Sci. Rep. 4, 5758 (2014).

30. Zhou, H., Cai, Y., Zhang, G. \& Zhang, Y. -W. Quantum thermal transport in stanene. Phys. Rev. B 94, 045423 (2016).

31. Cai, $\mathbf{X}$. et al. Sensitive room-temperature terahertz detection via the photothermoelectric effect in graphene. Nat. Nanotechnol. 9, 814-819 (2014).

32. Yan, J. et al. Dual-gated bilayer graphene hot-electron bolometer. Nat Nanotechnol. 7, 472-478 (2012)

33. Giovannetti, G. et al. Doping graphene with metal contacts. Phys. Rev. Lett. 101, 026803 (2008)

34. Xia, F., Perebeinos, V., Lin, Y. -M., Wu, Y. \& Avouris, P. The origins and limits of metal-graphene junction resistance. Nat. Nanotechnol. 6, 179-184 (2011).

35. Sakowicz, M. et al. Terahertz responsivity of field effect transistors versus their static channel conductivity and loading effects. J. Appl. Phys. 110, 054512 (2011).

36. Qin, H. et al. Room-temperature, low-impedance and high-sensitivity terahertz direct detector based on bilayer graphene field-effect transistor. Carbon 116 760-765 (2017).

37. Romeo, L. et al. Nanowire-based field effect transistors for terahertz detection and imaging systems. Nanotechnology 24, 214005 (2013).

38. Konstantatos, G. et al. Hybrid graphene-quantum dot phototransistors with ultrahigh gain. Nat. Nanotechnol. 7, 363-368 (2012). 